\title{
E3 Ubiquitin Ligase NEDD4 Regulates Estrogen Receptor Expression and Affects the Prognosis of Patients With Hormone Receptor-positive Breast Cancer
}

\author{
Yutaka Natori \\ Junko Suga \\ Fukushima Medical University \\ Emi Tokuda \\ Fukushima Medical University \\ Kazunoshin Tachibana \\ Fukushima Medical University \\ Jun-ichi Imai \\ Fukushima Medical University \\ Reiko Honma \\ Nippon Gene Co., Ltd. \\ Yusuke Azami \\ Fukushima Medical University \\ Masaru Noda \\ Fukushima Medical University \\ Eisaku Sasaki \\ Fukushima Medical University \\ Shinya Watanabe \\ Fukushima Medical University \\ Tohru Ohtake \\ Fukushima Medical University \\ Shigehira Saji ( $\nabla$ ss-saji@wa2.so-net.ne.jp ) \\ Fukushima Medical University
}

Fukushima Medical University https://orcid.org/0000-0002-9535-5298

\section{Research article}

Keywords: NEDD4, estrogen receptor, hormone receptor-positive breast cancer, hormone therapy, retrospective cohort study 
Posted Date: July 20th, 2021

DOI: https://doi.org/10.21203/rs.3.rs-713769/v1

License: (c) (i) This work is licensed under a Creative Commons Attribution 4.0 International License. Read Full License 


\section{Abstract}

Background: Neural precursor cell expressed developmentally downregulated 4-1 (NEDD4) is an E3 ligase that leads to the degradation of proteins including estrogen receptor a (ER). The E3 ligase can be a predictive marker of response to hormone therapy. We evaluated whether the expression level of NEDD4 affects the prognosis of hormone receptor-positive breast cancer patients. To support our clinical results, the association of NEDD4 with ER in breast cancer cells was studied.

Methods: We performed a retrospective cohort study enrolling 145 patients with hormone receptorpositive HER2-negative breast cancer with pathological stage 0 -II. Their expression levels of NEDD4 mRNA were measured, and disease-free survival (DFS) and overall survival (OS) were evaluated. Additionally, we knocked down NEDD4 in ER-positive breast cancer cells, MCF-7. We evaluated the expression level of ER in the cells and sensitivity to hormone therapy.

Results: Mean follow-up time was 6.2 years. Of 67 patients with high NEDD4 levels (high NEDD4 group) and 78 patients with low NEDD4 levels (low NEDD4 group), $97.0 \%$ and $94.9 \%$ of the patients received neoadjuvant/adjuvant hormone therapy, respectively. The hormone therapy included aromatase inhibitors and tamoxifen. DFS and OS of the low NEDD4 group were significantly longer than the high NEDD4 group ( $p=0.049$ and $p=0.023$ ). Western blot revealed high ER expression in NEDD4 knockdown cells. Proliferation of NEDD4 knockdown cells without estradiol stimulation as a simulation of the aromatase inhibitor therapy was suppressed to $53.6 \%$ of that with estradiol stimulation, whereas proliferation of NEDD4-expressing cells was suppressed to only 76.9\%. Proliferation of NEDD4 knockdown cells with exposure to estradiol and hydroxytamoxifen was suppressed to $57.9 \%$ of that without exposure to hydroxytamoxifen. In contrast, proliferation of NEDD4-expressing cells was suppressed to only $73.3 \%$. Thus, these simulations of hormone therapy showed a significant decrease in proliferation of NEDD4 knockdown cells.

Conclusion: Knockdown of NEDD4 in ER-positive breast cancer cells resulted in ER accumulation and high-sensitivity to hormone therapy. Hormone receptor-positive breast cancer patients with low expression of NEDD4 had favorable DFS and OS.

\section{Background}

Neural precursor cell expressed developmentally downregulated 4-1 (NEDD4), an E3 ubiquitin ligase, catalyzes ubiquitination of target proteins, leading to proteolysis of the target proteins in the proteasome [1]. NEDD4 targets several proteins involved in cancer growth, including RAS, AKT, and phosphatase and tensin homolog (PTEN) [2][3][4]. In breast cancer cells, NEDD4 promotes the degradation of human epidermal receptor (HER) 3, and it has been reported that defects in NEDD4 result in accumulation of HER3 [5]. With such accumulation of HER3, stimulation of breast cancer cells with neuregulin, a ligand for HER3, promotes proliferation of these cancer cells. The defect in NEDD4 resulting in HER3 accumulation had been expected to confer a worse clinical prognosis. On the contrary, a previous 
study reported that low expression of NEDD4 does not affect the clinical prognosis of breast cancer [6], and other studies reported that low expression of NEDD4 is associated with good prognosis of breast cancer [7][8].

Breast cancer is clinically categorized into three subtypes: HER2-positive breast cancer, in which HER2 is highly expressed; hormone receptor-positive breast cancer, in which estrogen receptor a (ER) and/or progesterone receptor are highly expressed; and triple-negative breast cancer, in which none of these three proteins are highly expressed. Each subtype is treated with its own therapy, and each has a different prognosis. Estradiol (E2), a ligand for ER, stimulates ER, and hormone receptor-positive breast cancer cells proliferate via nuclear translocation of ER and activation of tyrosine kinase signaling [9]. Hormone therapy targets ER to inhibit the growth of the hormone receptor-positive breast cancer cells. We previously reported that NEDD4 promotes the degradation of ER [10]. NEDD4 accelerates the degradation of ER, and therefore may affect the efficacy of hormone therapy and prognosis in hormone receptorpositive breast cancer patients. Prognosis of patients receiving perioperative neoadjuvant or adjuvant hormone therapy for 5-10 years is considered to be particularly affected by NEDD4.

In this study, we employed clinical and biological approaches to elucidate the prognostic impact of NEDD4 on hormone-receptor positive breast cancer. As a clinical approach, we retrospectively evaluated whether the expression level of NEDD4 affects the prognosis of hormone receptor-positive breast cancer patients. To reveal biological mechanisms that influenced the clinical results, as a biological approach, the association of NEDD4 with ER in breast cancer cells was also investigated.

\section{Material And Methods}

\section{Clinical sample material}

This study was approved by the ethics committee of Fukushima Medical University. Two hundred seventy-eight patients with breast cancer whose expression levels of NEDD4 mRNA were measured in Fukushima Medical University between February 2007 and November 2017 were enrolled in the study. To evaluate the simple association between prognosis of hormone receptor-positive breast cancer and NEDD4, hormone receptor-positive HER2-negative breast cancer cases were selected. Of these cases, 145 patients with pathological stage 0 -II for whom neoadjuvant/adjuvant hormone therapy for 5-10 years was recommended in the therapeutic guideline were included in the study[11]. We also included 51 hormone-receptor negative patients including HER2 positive and triple negative breast cancer patients. Clinical information was obtained from medical records in October 2019. Overall survival (OS) was defined as the time from the date of breast cancer diagnosis to the date of death from any cause. Disease-free survival (DFS) was defined as the time from the date of surgery to the date of recurrence or death, whichever occurred first. Patients in whom no event was observed were censored on the day of documentation of last medical records.

\section{NEDD4 mRNA expression analysis}


Breast cancer tissues were obtained from surgical or biopsy specimens. The specimens were frozen and processed for total RNA extraction using Isogen (Nippon Gene Co., Ltd., Tokyo, Japan) and for poly(A)+RNA purification using MicroPoly(A) Purist kit (Ambion, Austin, TX, USA) [12]. The DNA microarray that was used for the poly $(A)+R N A$ was named system 1 , in which a set of synthetic polynucleotides (80-mers) representing 31,797 species of human transcript sequences including NEDD4 was printed on a glass slide using a custom arrayer. The DNA microarray that was used for total RNA was named System 2, in which a set of synthetic polynucleotides (80-mers) representing 14,400 species of human transcript sequences including NEDD4 was printed on a glass slide using a custom arrayer. For the RNA of the samples, SuperScript II (Invitrogen Life Technologies, Carlsbad, CA, USA) and Cyanine 5dUTP (Perkin-Elmer Inc., Boston, MA, USA) were used to synthesize labeled cDNA from $2 \mu \mathrm{g}$ of poly $(A)+R N A$ in System 1, and $5 \mu \mathrm{g}$ of total RNA in System 2. Human common reference RNA was prepared by mixing equal amounts of total RNA and poly $(A)+R N A$, which were extracted from 22 human cancer cell lines (A431, A549, Akl, hBL-100, HeLa, hepG2, hL60, ImR-32, Jurket, k562, kP4, mkN7, Nk-92, Raji, Rd, Saos-2, Sk-N-mC, SW-13, T24, U251, U937, and Y79). Using a same method for the human common reference RNA, Cyanine 3-dUTP (Perkin-Elmer Inc.) was used to synthesize labeled cDNA from 2 $\mu \mathrm{g}$ of Human Universal Reference RNA Type I (MicroDiagnostic, Tokyo, Japan) in System 1, and $5 \mu \mathrm{g}$ of Human Universal Reference RNA Type II (MicroDiagnostic) in System 2. Hybridization was performed with a Labeling and Hybridization kit (MicroDiagnostic). Signals were measured using a GenePix 4000B Scanner (Axon Instruments, Inc., Union City, CA, USA) and then converted into primary expression ratios of the cyanine 5 intensity of each specimen to the cyanine 3 intensity of the Human Universal Reference RNA. Each ratio was normalized using GenePix Pro 3.0 software (Axon Instruments, Inc.). The primary expression ratios were converted into log2 values, which were designated as converted values. The converted log2 values of NEDD4 mRNA was used in the present study.

\section{Cell culture}

ER-positive human breast cancer cell line, MCF-7, and human embryonic kidney cell line, 293T, were purchased from ATCC (Manassas, VA, USA). The cells were cultured in Dulbecco's modified Eagle's medium (DMEM, Fujifilm Wako, Osaka, Japan) supplemented with 10\% heat-inactivated fetal bovine serum (FBS, Biowest, Nuaille, France), 100 units $/ \mathrm{ml}$ penicillin $\mathrm{G}$ and $100 \mu \mathrm{g} / \mathrm{ml}$ streptomycin in a humidified $5 \% \mathrm{CO} 2$ incubator at $37^{\circ} \mathrm{C}$. As pretreatment for evaluating the cells with $\mathrm{E} 2$ stimulation or hydroxytamoxifen (TAM) exposure, the MCF-7 cells were cultured for two days in phenol red-free DMEM (Fujifilm Wako) containing 10\% heat-inactivated FBS stripped of steroids by absorption to dextran-coated charcoal FBS (Biological Industries, Beit HaEmek, Israel). The MCF-7 cells were stimulated by $1 \mathrm{nM}$ E2 (17b-estradiol, Sigma-Aldrich, St. Louis, MO, USA) for 48 hours. When simulating clinical tamoxifen therapy, the MCF-7 cells were exposed to 1 nM E2 + 2 mM TAM (4-Hydroxytamoxifen, Sigma-Aldrich) for 48-72 hours.

\section{Small hairpin RNA (shRNA) mediated knockdown}


Lentivirus vector expressing shRNA for NEDD4 (sh-NEDD4) was constructed in pRSI12-U6-sh-HTS4-UbiCTagRFP-2A-Puro plasmid (Cellecta, Mountain View, CA, USA). Lentivirus vector expressing shRNA for no target genes (sh-control) was also constructed in the plasmid. The sh-NEDD4 or the sh-control was transfected into 293T cells together with two packaging plasmids, pCMV-VSV-G/RSV-Rev and pCAGHIVgp (RIKEN Bio-Resource Center, Tsukuba, Japan), using FuGENE HD (Promega, Madison, WI, USA). At 48 hours post-transfection, the supernatants were collected and filtered. The lentivirus encoding the shRNA were incubated with MCF-7 cells for $48 \mathrm{~h}$. The transduced cells were selected for additional incubation for $72 \mathrm{~h}$ in the presence of $1 \mu \mathrm{g} / \mathrm{ml}$ puromycin (Fujifilm Wako).

\section{Cell proliferation assay}

The sh-control or sh-NEDD4 knockdown MCF-7 cells were seeded in 96-well culture plates $\left(7 \times 10^{3}\right.$ cells/well). After overnight incubation, the cells were replaced in a medium containing ethanol or $1 \mathrm{nM}$ E2 or $1 \mathrm{nM}$ E2 + 2 mM TAM. After incubation, a Cell Counting Kit-8 (Dojindo, Kumamoto, Japan) solution was added to the wells. After incubating the cells for 2 hours at $37^{\circ} \mathrm{C}$, absorbance was measured using a plate reader (Thermo Fisher Scientific, Waltham, MA, USA). The absorbance was proportional to cell proliferation.

\section{Western Blots}

Western blot samples were prepared from the cultured cells that were lysed in RIPA buffer (40 mM Tris$\mathrm{HCl}, \mathrm{pH} 7.5,1 \% \mathrm{NP}-40,150 \mathrm{mM} \mathrm{NaCl}, 2 \mathrm{mM}$ EDTA, $2 \mathrm{mM}$ Na3V04, $50 \mathrm{mM} \mathrm{NaF}$ ) containing protease inhibitor cocktail (Roche, Basel, Swiss). The samples were boiled for $5 \mathrm{~min}$ at $95^{\circ} \mathrm{C}$. Lysates were centrifuged at $13,000 \mathrm{~g}$ for $20 \mathrm{~min}$ at $4^{\circ} \mathrm{C}$. Proteins in the samples were separated in $4-20 \%$ sodium dodecyl sulfate-polyacrylamide gel electrophoresis (Bio-Rad, Hercules, CA, USA), and transferred to polyvinylidene difluoride membranes (Merck Millipore, Burlington, MA, USA). The proteins were blotted with primary and secondary antibodies. The antibodies used were as follows: NEDD4, HER3, PTEN, phospho-HER3 (pHER3[Y1289]), RAS, ERK1/2, phospho-ERK1/2 (pERK1/2[T202/Y204]), AKT, phosphoAKT (pAKT[S473]) (Cell Signaling Technology, Danvers, MA, USA), ER (Thermo Fisher Scientific) and $\beta$ actin (Sigma-Aldrich). Expression levels of the proteins were visualized by SuperSignal West Pico PLUS chemiluminescent substrate (Thermo Fisher Scientific) and ChemiDoc XRS Plus (Bio-Rad).

\section{Statistics analysis}

All of the analyses, including receiver operating characteristic (ROC) curve analysis, Kaplan-Meier survival analysis log-rank test, and Student's t-test, were conducted using $\mathrm{R}$ version 4.0.3. Kaplan-Meier survival analysis and the log-rank test were used to compare differences in OS or DFS for each clinical categorical variable. The cell proliferation assays were expressed as means \pm standard deviation. Statistical analysis of the cell proliferation assay was performed using the Student's t-test (two-tail). P-values $<0.05$ were considered statistically significant.

\section{Results}


Low NEDD4 mRNA level is associated with a good prognosis in hormone receptor-positive breast cancer

As the expression level of NEDD4 protein in breast cancer cells was reported to be correlated with that of NEDD4 mRNA, the NEDD4 mRNA level was used as an indicator of NEDD4 protein level in the present study [13]. Expression levels of NEDD4 mRNA were measured in the cancer tissues from a total of 145 patients with hormone receptor-positive HER2-negative breast cancer pathological stage $0-$ II. An ROC curve was plotted from the NEDD4 mRNA levels and DFS data (Supplemental data 1). The cut-off level at which sensitivity and specificity were maximized was calculated from the ROC curve as -0.495 . Of all 145 patients, 67 who had NEDD4 mRNA levels $\geq-0.495$ were classified into the high NEDD4 group, and 78 who had levels $<-0.495$ were classified into the low NEDD4 group (Table 1). All cases were Japanese women and had undergone breast cancer surgery. The median ages at diagnosis were 54 years (range 30-85 years) and 61 years (range 29-85 years) in the high NEDD4 and low NEDD 4 groups, respectively. The mean follow-up duration was 6.2 years (range 1 month-12 years). The proportions of pathological stages of both groups were almost the same; $1.5 \%$ stage $0,38.8 \%$ stage I, and $59.7 \%$ stage II in the high NEDD4 group, $1.3 \%$ stage $0,44.9 \%$ stage I, and $53.8 \%$ stage II in the low NEDD4 group.

Neoadjuvant/adjuvant hormone therapy was used in $97.0 \%$ of the high NEDD 4 group and $94.9 \%$ of the low NEDD 4 group. Of the high NEDD4 group, 53.7\% used aromatase inhibitor (anastrozole and letrozole) and $43.3 \%$ used tamoxifen, while $69.2 \%$ and $25.6 \%$ of the low NEDD 4 group used them, respectively. Luminal subtypes were not classified due to the lack of Ki67 data. The Kaplan-Meier survival (DFS and OS) analysis and log-rank test were used to compare survival differences between the high NEDD4 and low NEDD4 groups (Figure 1). The low NEDD4 group had significantly longer DFS than the high NEDD4 group ( $p=0.049)$, and had also significant longer OS $(p=0.023)$. we also examined the correlation between the expression level of NEDD4 and prognosis. Group with extremely high expression of NEDD4 mRNA ( $\geq 0.200$ ) had much shorter DFS and OS (Supplemental data 2). According to the univariate log-rank analyses, conventional prognostic factors (age, lymph node metastasis, and clinical stage) did not show statistical significance for DFS or OS in this study population (Table 2). Therefore, multivariate analysis was not performed. We also evaluated prognostic impact of NEDD4 on the 51 hormone-receptor negative breast cancer patients with pathological stage 0-II including HER2-positive or triple-negative cancer. In the same way as with the 145 patients, the 51 patients were divided into two groups using the NEDD4 mRNA levels $\geq-0.495(n=13)$ and $<-0.495(n=38)$. Kaplan-Meier curves showed that NEDD4 level was not a prognostic indicator for DFS and OS in these patients (Supplemental data 3).

\section{NEDD4 knockdown increases ER level and E2-stimulated ERK signaling}

The effect of NEDD4 knockdown on the hormone receptor-positive breast cancer cells was examined using the ER-positive breast cancer cell line, MCF-7. NEDD4 in the MCF-7 cells was knocked down by the sh-NEDD4 (Figure 2A). Western blot of the sh-NEDD4 cells showed high expression of ER and HER3, but no change in RAS, AKT, or PTEN. $b$ actin was used as an internal control. ER is well-known to be degraded by E2 stimulation in the proteasome system [14]. In the present study, a decrease in expression of ER in MCF-7 cells stimulated by $1 \mathrm{nM}$ E2 was validated (Figure 2B). It is documented that stimulated ER 
activates downstream ERK and PI3K/AKT signaling pathways [9]. In the sh-NEDD4 cells stimulated with 1nM E2, an increase in ERK1/2 (T202/Y404) phosphorylation was observed; however, AKT (S473) phosphorylation was not increased (Figure 2C). HER3 (Y1289) was not phosphorylated at any condition.

\section{NEDD4 knockdown enhances suppression of cell growth by hormone therapy}

Aromatase inhibitors reduce the production of E2 by inhibiting its synthesis in a human body. The effect of reducing E2 by the aromatase inhibitors in a human body can be mimicked as the absence of E2 stimulation to cultured breast cancer cells. In the sh-control cells and the sh-NEDD4 cells, 1 nM E2 stimulation increased cell proliferation by $40 \%$ and $161 \%$, respectively, at 48 hours post-stimulation (Figures 3B). However, no difference in proliferation of those cells was shown in the absence of the E2 stimulation (Figure 3A). Cell proliferation of the sh-NEDD4 cells without E2 stimulation at 48 hours was suppressed to $53.6 \%$ of that with E2 stimulation (Figure 3D and 3E). On the other hand, proliferation of the sh-control cells was suppressed to only $76.9 \%$ (Figures $3 \mathrm{C}$ and $3 \mathrm{E}$ ). Absence of E2 stimulation significantly inhibited cell proliferation of the sh-NEDD4 cells compared to the sh-control cells $(p=7.3 \times$ $10^{-4}$ ). We also examined the inhibitory effect of TAM on cell proliferation. Cell proliferation of the shNEDD4 cells with exposure to $1 \mathrm{nM}$ E2 $+2 \mathrm{mM}$ TAM at 72 hours was suppressed to $57.9 \%$ (Figures $3 \mathrm{G}$ and $3 \mathrm{H}$ ), however, proliferation of the sh-control cells was suppressed to only $73.3 \%$ (Figure $3 \mathrm{~F}, 3 \mathrm{H})$. The TAM treatment significantly inhibited cell proliferation in the sh-NEDD4 cells compared to the sh-control cells $(p=0.041)$.

\section{Discussion}

NEDD4 is an E3 ubiquitin ligase that ubiquitinates and leads to the degradation of various proteins in the proteasome [1]. ER is ubiquitinated by E3 ubiquitin ligases such as MDM2 and BRCA1, and is degraded in the proteasome [15]. In our previous study, we demonstrated that NEDD4 was a novel E3 ligase associated with ER degradation [10]. In the present study, we found that the knockdown of NEDD4 leads to high expression of ER, suggesting that ER accumulation occurs due to the disorder of the degradation system. Proliferation of the NEDD4 knockdown cells was significantly inhibited in the absence of E2 stimulation compared to the control cells. Cell culture in the absence of E2 stimulation simulates the depletion of E2 in human bodies treated with aromatase inhibitors. Thus, the significant decrease in proliferation of NEDD4 knockdown cells in the absence of E2 stimulation suggests that aromatase inhibitors have high efficacy on the same cells in human bodies. Favorable DFS and OS of the low NEDD4 group in our cohort study could have reflected high efficacy of the hormone therapy including aromatase inhibitors on the low NEDD4 breast cancer. According to the current therapeutic guidelines, early-stage hormone receptor-positive breast cancer patients should receive neoadjuvant/adjuvant hormone therapy (aromatase inhibitors or tamoxifen) for about 5-10 years [11]. In the present cohort study, about 53.7-69.2\% of the patients were treated with aromatase inhibitors (anastrozole and letrozole) as the neoadjuvant/adjuvant hormone therapy. This cohort study demonstrated that the low NEDD4 group had significantly longer DFS and OS than the high NEDD4 group. Our clinical and 
biological approaches suggest that ER accumulation in hormone receptor-positive breast cancer with low NEDD4 expression results in significant sensitivity to aromatase inhibitors, and prolongs prognosis.

A similar pattern was observed for tamoxifen treatment. In the biological simulation of the tamoxifen treatment, NEDD4 knockdown cells were also more suppressed than NEDD4-expressing cells. In our clinical approach in the present study, about $25.6-43.3 \%$ of the patients were treated with tamoxifen. The ER accumulation caused by low NEDD4 expression could lead to high sensitivity to the tamoxifen treatment. In summary, low expression of NEDD4 in hormone receptor-positive HER2-negative breast cancer causes accumulation of ER, which leads to a favorable response to hormone therapy and prolonged prognosis. The results of the present study suggests that low expression of NEDD4 would be a predictive marker of response to hormone therapy.

Another research group reported that prognosis of hormone-receptor positive breast cancer was independent of the expression level of NEDD4, while prognosis of hormone-receptor negative breast cancer was dependent of that level [8]. The prognoses were compared using the web-based database of Kaplan-Meier plotter. In the database, patients' characteristics including hormone therapy was not clear, and the cut-off value of NEDD4 level for dividing the patients into two groups was also not clear. Thus, the prognosis among the breast cancer subtypes differs from our results, but the poor prognosis of high expression of NEDD4 is consistent with our result. NEDD4 has many substrates for degradation. However, expression levels of RAS, AKT, and PTEN, which have been reported to be increased by NEDD4 knockdown, did not change in the present study [1][2][4]. There are contrary reports showing that NEDD4 does not involve PTEN degradation [13][16]. NEDD4-mediated ubiquitination regulates the levels of phosphorylated AKT but not total AKT [3][17]. As the degradation of RAS via the NEDD4 ubiquitination system is regulated by negative feed-back regulation induced by the signaling of RAS itself, there is no change in degradation without RAS signaling [2]. Thus, the expression levels of RAS, AKT and PTEN in NEDD4 knockdown cells have been controversial. In the present study, we think that the high sensitivity to hormone therapy is independent of RAS, AKT, and PTEN because there is no change in the expression levels of these proteins in NEDD4 knockdown cells. Although NEDD4 knockdown caused accumulation of HER3, it was not enough to activate HER3. This result is similar to that of a previous report that HER3 is not activated and does not lead to cell proliferation without a ligand [5].

As a limitation of this study, we have not proved ubiquitination of ER mediated by NEDD4 followed by degradation of ER in proteasomes. Although we demonstrated the inverse correlation between the level of NEDD4 and ER, further study is required to elucidate the interaction of these two proteins. As this retrospective cohort study had group biases including age and drugs of hormone therapy, a prospective study is required for statistical accuracy in future.

\section{Conclusion}

This article is the first to address that NEDD4 regulating expression of ER influences the prognosis of hormone-positive breast cancer patients. We here showed that high expression of ER in hormone 
receptor-positive breast cancer cells via NEDD4 knockdown leads to high sensitivity to hormone therapy. In the retrospective cohort study, hormone receptor-positive HER2-negative breast cancer patients with low NEDD4 level had a good prognosis. NEDD4 expression is a predictive marker of response to hormone therapy.

\section{Abbreviations}

NEDD4, neural precursor cell expressed developmentally downregulated $4-1$; ER, estrogen receptor a; DFS, disease-free survival; OS, overall survival; E2, estradiol; TAM, hydroxytamoxifen; PTEN, phosphatase and tensin homolog; HER, human epidermal receptor; DMEM, Dulbecco's modified Eagle's medium; FBS, fetal bovine serum; shRNA, small hairpin RNA, sh-NEDD4, lentivirus vector expressing shRNA for NEDD4; sh-control, lentivirus vector expressing shRNA for no target genes ; pHER3(Y1289), phospho-HER3; pERK1/2(T202/Y204), phospho-ERK1/2; pAKT(S473), phospho-AKT; ROC, receiver operating characteristic

\section{Declarations}

\section{Acknowledgements}

We thank Dr. Yuko Murakami for collection of the clinical data; Dr. Emi Ito, Dr. Hirosumi Tamura, and Masae Ryufuku for the mRNA analysis.

\section{Author's contributions}

YN and SS designed the study. YN and ES performed the statistical analyses. YN and JS conducted the Western blots and the cell-proliferation assays. YN, ET, KT, YA, MN, and TO collected the clinical information. JI, RH and SW conducted the mRNA analysis. YN prepared the original draft with suggestions from the other authors. All authors interpreted the results, and read and approved the final manuscript.

\section{Funding}

This work was supported by grant of Japan Society for the Promotion of Science KAKENHI, number JP19K09079.

\section{Availability of data and materials}

The datasets generated and/or analyzed during the current study are available from the corresponding author on reasonable request.

\section{Ethics approval and consent to participate}


This study was approved by the ethics committee of Fukushima Medical University. The consent for the mRNA analysis was in the patients' consent forms.

\section{Consent for publication}

Not applicable.

\section{Competing interests}

Saji reports grants-in-aid for scientific research from Japan Society for the Promotion of Science KAKENHI, number JP19K09079, Radiation Disaster and Medical Science Research Center Triangle Project, Taiho, Eisai, Chugai, Takeda, MSD, Novartis, Astra Zeneca, and Daiichi Sankyo. Saji reports personal fees (lecture fee) from Chugai, Kyowa Kirin, MSD, Novartis, Eisai, Takeda, Daiichi Sankyo, Eli Lilly, Astra Zeneca, Pfizer, Taiho and Nihonkayaku outside the submitted work, and is an executive board member of JBCRG and JBCS. Tokuda reports grants-in-aid for scientific research from Japan Society for the Promotion of Science KAKENHI, number JP21K08599 and Eli Lilly Japan. Tokuda reports personal fees from Chugai, Pfizer, Novartis and Daiichi Sankyo outside the submitted work.

\section{Author details}

${ }^{1}$ Department of Medical Oncology, Fukushima Medical University, Fukushima, Japan. ${ }^{2}$ Department of Breast Surgery, Fukushima Medical University, Fukushima, Japan. ${ }^{3}$ Translational Research Center, Fukushima Medical University, Fukushima, Japan. ${ }^{4}$ Nippon Gene Co., Ltd., Tokyo, Japan

\section{References}

1. Huang X, Chen J, Cao W, Yang L, Chen Q, He J, et al. The many substrates and functions of NEDD4-1. Cell Death Dis. 2019;10:904.

2. Zeng T, Wang Q, Fu J, Lin Q, Bi J, Ding W, et al. Impeded Nedd4-1-Mediated Ras Degradation Underlies Ras-Driven Tumorigenesis. Cell Rep. 2014;7:871-82.

3. Fan CD, Lum MA, Xu C, Black JD, Wang X. Ubiquitin-dependent regulation of phospho-AKT Dynamics by the ubiquitin E3 LIGASE, NEDD4-1, in the insulin-like growth factor-1 response. J Biol Chem. 2013;288:1674-84.

4. Wang X, Trotman LC, Koppie T, Alimonti A, Chen Z, Gao Z, et al. NEDD4-1 Is a Proto-Oncogenic Ubiquitin Ligase for PTEN. Cell. 2007;128:129-39.

5. Huang Z, Choi BK, Mujoo K, Fan X, Fa M, Mukherjee S, et al. The E3 ubiquitin ligase NEDD4 negatively regulates HER3/ErbB3 level and signaling. Oncogene. 2015;34:1105-15.

6. Luhtala S, Staff S, Kallioniemi A, Tanner M, Isola J. Clinicopathological and prognostic correlations of HER3 expression and its degradation regulators, NEDD4-1 and NRDP1, in primary breast cancer. BMC Cancer. 2018;18:1045. 
7. Wan L, Liu T, Hong Z, Pan Y, Sizemore ST, Zhang J, et al. NEDD4 expression is associated with breast cancer progression and is predictive of a poor prognosis. Breast Cancer Res Breast Cancer Research. 2019;21:148.

8. Jeon S-A, Kim DW, Lee D-B, Cho* J-Y. NEDD4 Plays Roles in the Maintenance of Breast Cancer Stem Cell Characteristics. Front Oncol. 2020;10:1-12.

9. Alfakeeh A, Brezden-Masley C. Overcoming endocrine resistance in hormone receptor-positive breast cancer. Curr Oncol. 2018;25:18-27.

10. Suga J, Izumiyama K, Tanaka N, Saji S. Estradiol promotes rapid degradation of HER3 in ER-positive breast cancer cell line MCF-7. Biochem Biophys Reports. 2018;16:103-9.

11. NCCN guigelines Breast Cancer. 2021. https://www.nccn.org/guidelines/guidelines-detail? category=1\&id=1419. Accessed 18 June 2021.

12. Tada T, Honma R, Imai JI, Saze Z, Kogure M, Marubashi S, et al. A novel gene expression scoring system for accurate diagnosis of basaloid squamous cell carcinoma of the esophagus. Int $\mathrm{J}$ Oncol. 2017; 51:877-86.

13. Chen Y, van de Vijver MJ, Hibshoosh H, Parsons R, Saal LH. PTEN and NEDD4 in Human Breast Carcinoma. Pathol Oncol Res. 2016;22:41-7.

14. Nawaz Z, Lonard DM, Dennis AP, Smith CL, O'Malley BW. Proteasome-dependent degradation of the human estrogen receptor. Proc Natl Acad Sci. 2002;96:1858-62.

15. Zhou W, Slingerland JM. Links between oestrogen receptor activation and proteolysis: Relevance to hormone-regulated cancer therapy. Nat Rev Cancer. 2014;14:26-38.

16. Fouladkou F, Landry T, Kawabe H, Neeb A, Lu C, Brose N, et al. The ubiquitin ligase Nedd4-1 is dispensable for the regulation of PTEN stability and localization. Proc Natl Acad Sci. 2008;105:8585-90.

17. Zhang Y, Goodfellow R, Li Y, Yanga S, Winters CJ, Thiel KW, et al. NEDD4 ubiquitin ligase is a putative oncogene in endometrial cancer that activates IGF-1R/PI3K/Akt signaling. Gynecol Oncol. 2015;139:127-33.

\section{Tables}

Table 1. Clinicopathological characteristics of the patients with hormone receptor-positive HER2-negative breast cancer, classified into high- and low-NEDD4 mRNA groups 
Characteristics

\begin{tabular}{lll} 
Age median (range) & $54(30-85)$ & $61(29-85)$ \\
\hline Female (\%) & $67(100)$ & $78(100)$ \\
\hline Stage (\%) & & $1(1.3)$ \\
\hline 0 & $1(1.5)$ & $35(44.9)$ \\
\hline I & $26(38.8)$ & $42(53.8)$ \\
\hline II & $40(59.7)$ & $26(33.3)$ \\
\hline Lymph node metastasis (\%) & $23(34.3)$ & $78(100)$ \\
\hline Estrogen receptor (+) (\%) & $67(100)$ & $68(87.2)$ \\
\hline Progesterone receptor (+) (\%) & $48(71.6)$ & $0(0)$ \\
\hline HER2 (+) (\%) & $0(0)$ & $78(100)$ \\
\hline Surgery (\%) & $67(100)$ & $21(26.9)$ \\
\hline Chemotherapy (\%) & $27(40.3)$ & $3(3.8)$ \\
\hline Neoadjuvant & $2(3.0)$ & $18(23.1)$ \\
\hline Adjuvant & $25(37.3)$ & $38(48.7)$ \\
\hline Radiotherapy (\%) & $41(61.2)$ & $74(94.9)$ \\
\hline Neoadjuvant/adjuvant hormone therapy (\%) & $65(97.0)$ & $54(69.2)$ \\
\hline Aromatase inhibitors & $36(53.7)$ & $20(25.6)$ \\
\hline Tamoxifen & $29(43.3)$ & $-0.782(-1.681--0.496)$ \\
\hline NEDD4 mRNA (range) & $-0.250(-0.494-1.105)$ & $(\mathrm{s})$ \\
\hline
\end{tabular}

Table 2. Univariate log-rank analyses for prognostic factors and NEDD4 mRNA level of hormone receptorpositive HER2-begative breast cancer. $P$-values which are statistically significant are marked with asterisk $(*)$. 


\section{Univariate analysis}

\begin{tabular}{lll}
\hline & DFS & OS \\
\hline Age $>50$ vs $<50$ & 0.880 & 0.621 \\
\hline Lymph node metastasis (+) vs $(-)$ & 0.591 & 0.947 \\
\hline Stage II vs $0-$ I & 0.057 & 0.759 \\
\hline Chemotherapy (+) vs (-) & 0.590 & 0.908 \\
\hline Radiotherapy (+) vs (-) & 0.978 & 0.571 \\
\hline high NEDD4 vs low NEDD4 & $0.049 *$ & $0.023^{*}$
\end{tabular}

\section{Figures}

A

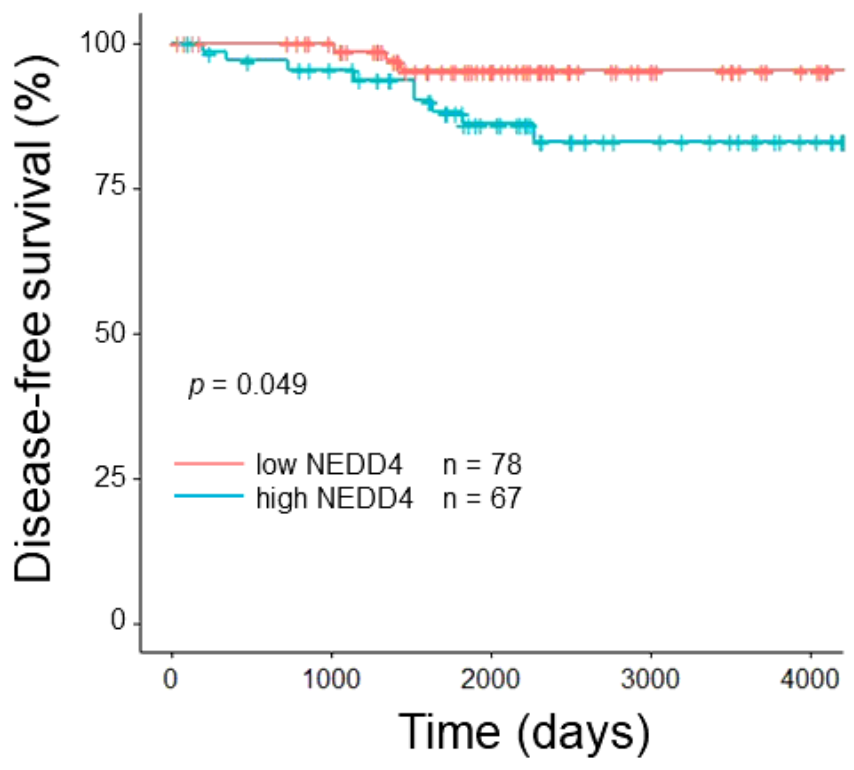

B

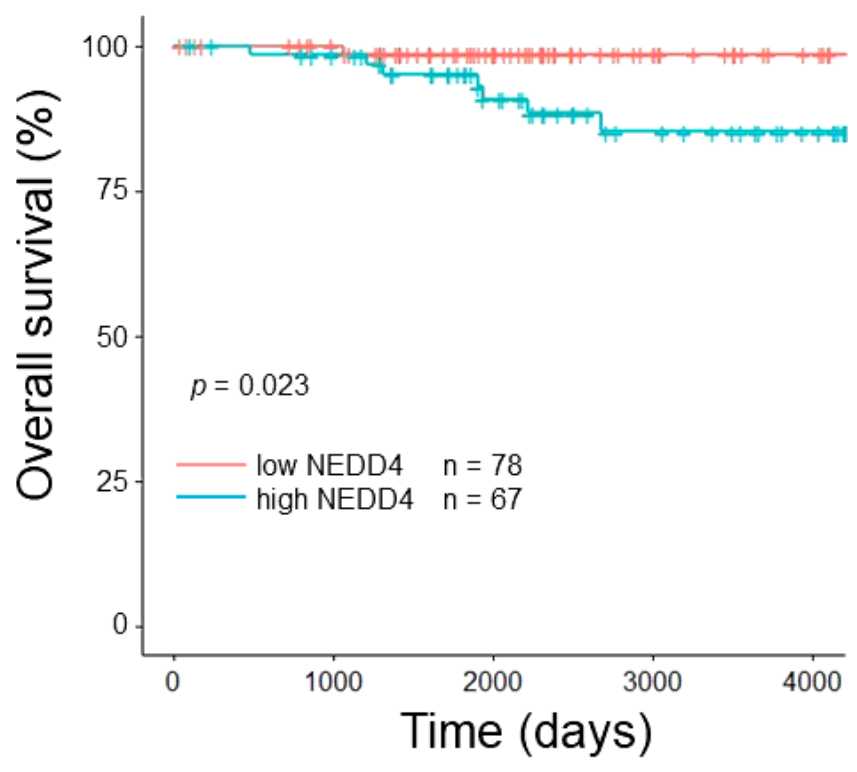

\section{Figure 1}

Prognostic impact of NEDD4 on hormone receptor-positive HER2-negative breast cancer patients. (A) Kaplan-Meier curves for DFS showing a good prognosis in low NEDD4 group $(p=0.049)$. (B) KaplanMeier curves for OS showing a good prognosis in low NEDD4 group $(p=0.023)$. Log rank (Mantel-Cox $) p-$ values $<0.05$ were considered sta-tistically significant. 


\section{$\underline{\text { MCF-7 }}$}

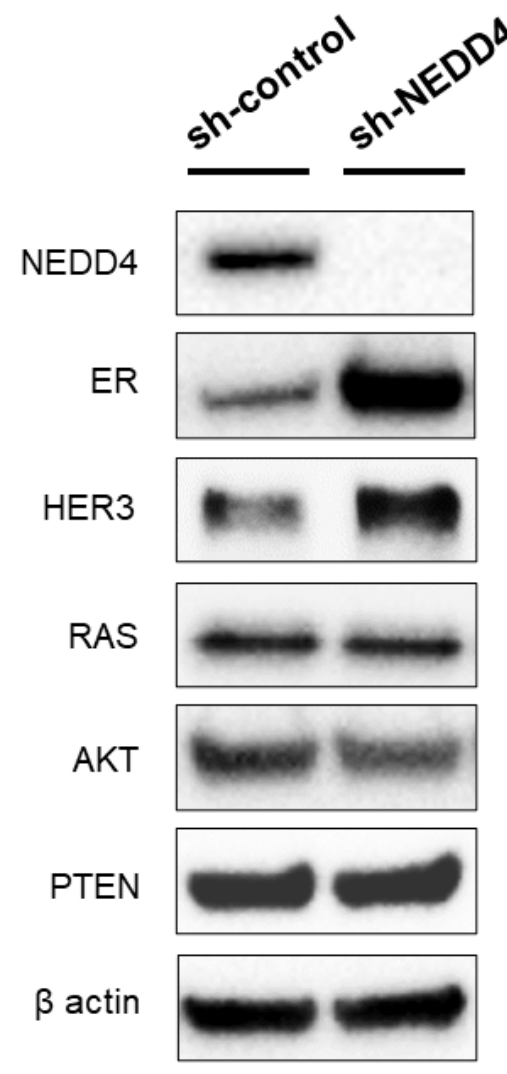

B

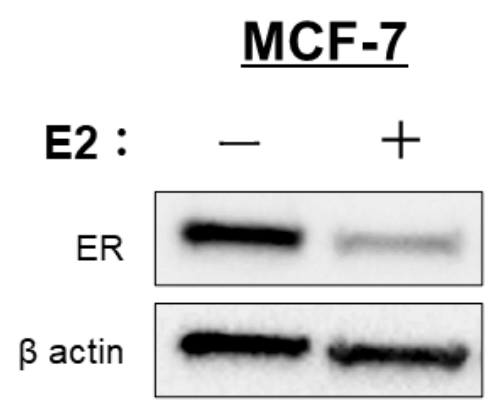

$\underline{\text { MCF-7 }}$

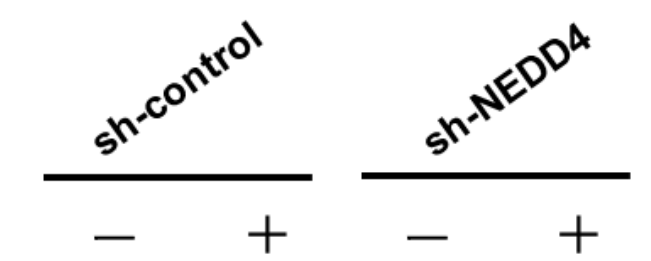

E2 :

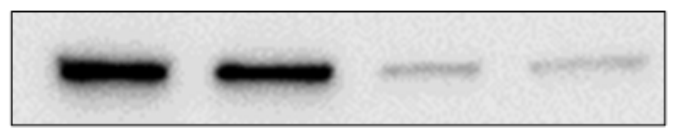

NEDD4

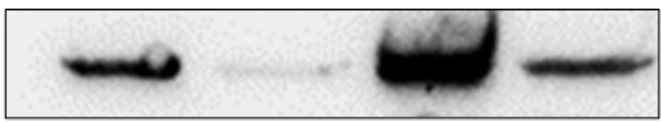

HER3

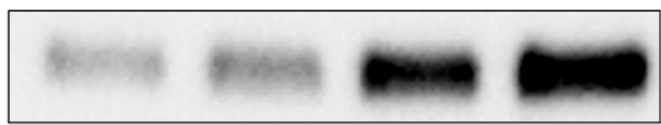

pHER3(Y1289)

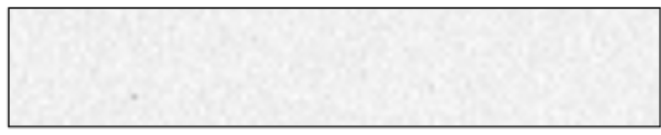

ERK1/2

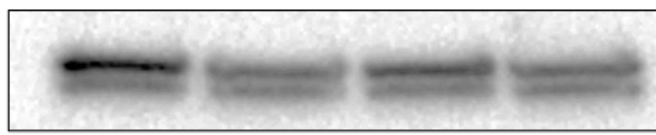

pERK1/2(T202/Y204)

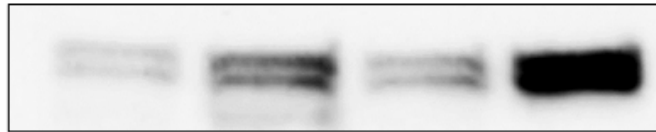

AKT

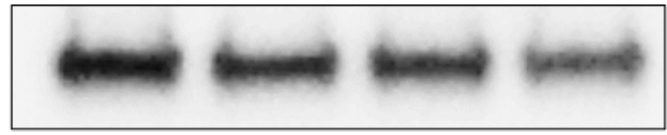

pAKT(S473)

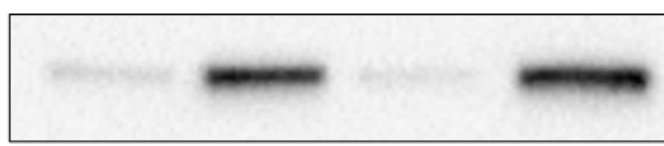

$\beta$ actin

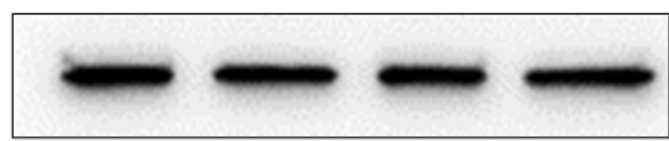

\section{Figure 2}

NEDD4 knockdown increased the expression level of ER and E2-stimulated phosphory-lation of ERK1/2. Whole cell lysates of MCF-7 cells were subjected to Western blotting. (A) Expression levels of NEDD4 and proteins associated with NEDD4 in sh-control and sh-NEDD4 cells. Western blotting showed no expression of NEDD4 and high expression of ER and HER3 in the sh-NEDD4 cells. (B) Western blotting of MCF-7 cells stimulated with $1 \mathrm{nM}$ E2 showed degradation of ER. (C) Phosphorylation of ERK1/2 (pERK1/2[T202/Y204]) and AKT (pAKT[S473]) stimulated by 1nM E2 were observed in the sh-control, and 
sh-NEDD4 cells. The phosphorylation of ERK1/2 in the sh-NEDD4 cells was greater than that of the shcontrol cells.

A

MCF-7

E2（-)

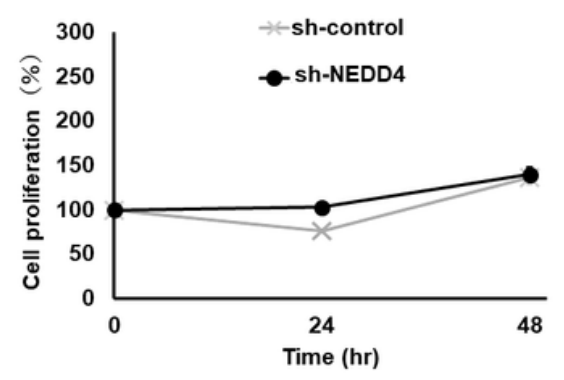

C

MCF-7

sh-control

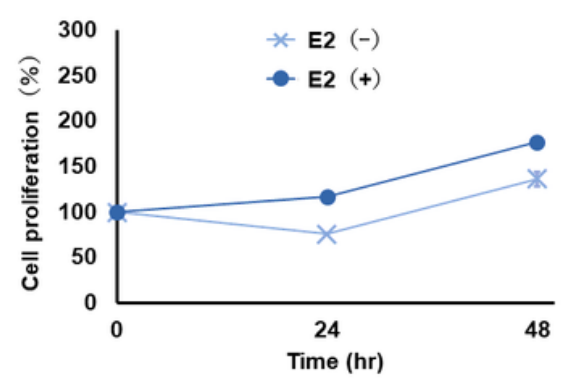

$\mathbf{F}$

MCF-7

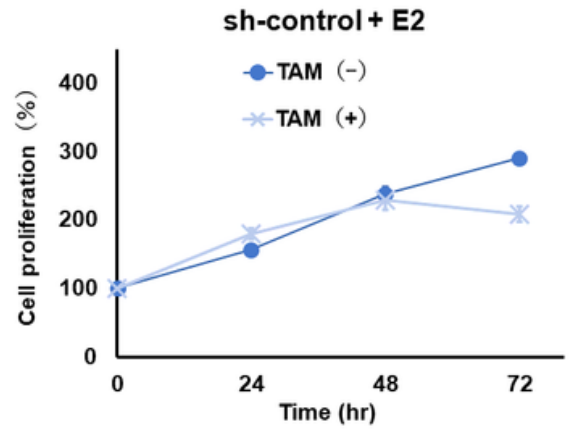

B

MCF-7

E2（+）

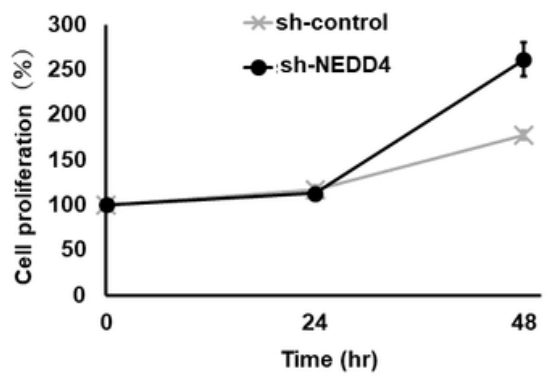

D

MCF-7

sh-NEDD4

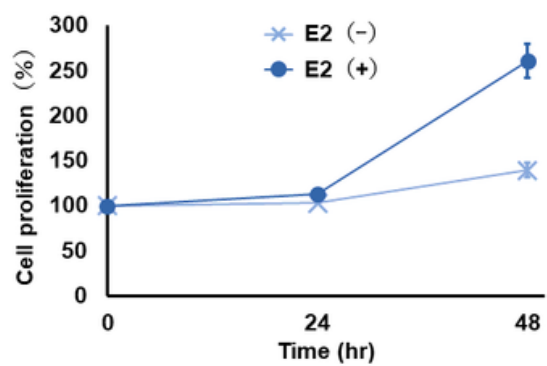

G

\section{MCF-7}

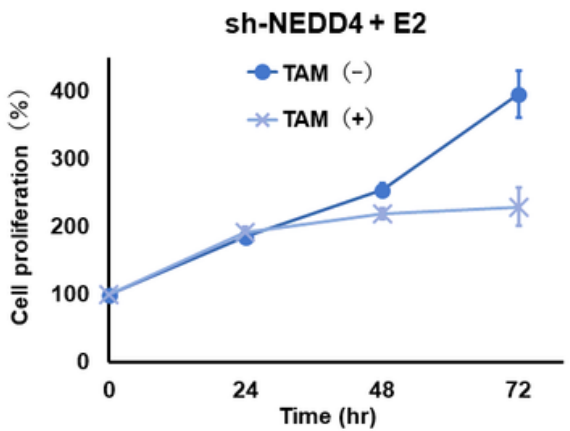

E

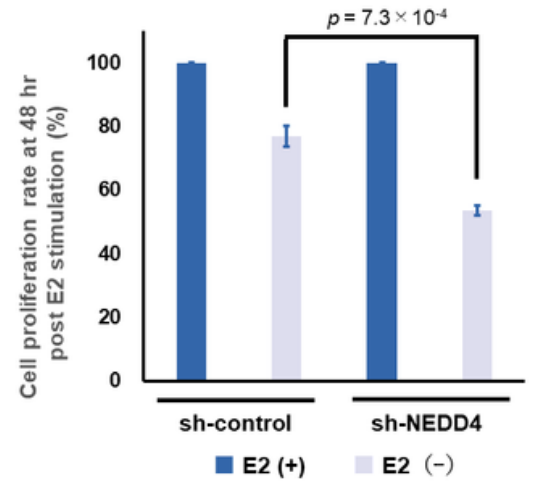

H

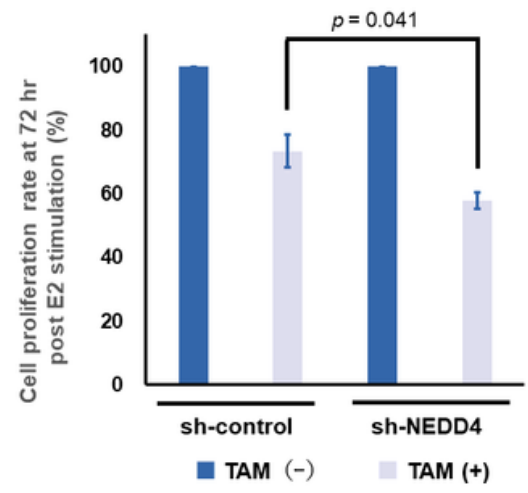

Figure 3

NEDD4 knockdown enhanced E2-dependent cell proliferation and sensitivity to TAM. Cell proliferation was measured using the Cell Counting Kit-8. (A-D) Proliferation of the sh-control and sh-NEDD4 cells with or without $1 \mathrm{nM}$ E2 stimulation. (E) Comparison of cell proliferation rate of the sh-control and shNEDD4 cells at 48 hours with or without E2 stimulation. $(F, G)$ E2-stimulated cell proliferation of the shcontrol and sh-NEDD 4 cells with or without $2 \mu \mathrm{M}$ TAM exposure. $(\mathrm{H})$ Comparison of E2-stimulated cell prolif-eration rate of the sh-control and sh-NEDD4 cells at 72 hours with or without $2 \mu \mathrm{M}$ TAM exposure. 


\section{Supplementary Files}

This is a list of supplementary files associated with this preprint. Click to download.

- Suppl1NEDD4natori.tif

- SuppI2NEDD4natori.tif

- SuppI3NEDD4natori.tif 